\title{
Clinical Aspects of Direct Digital Mammography
}

\author{
G.J.S. Parkin
}

Since 1990, computed radiography (CR) has been used routinely in our symptomatic mammography service, imaging approximately 2,000 patients per year. Careful selection of the appropriate image processing parameters results in high-quality images of diagnostic value equivalent to conventional film-screen mammograms. Problems encountered included dust artifacts, black films, and white films, but these constituted only a very small proportion of images obtained and the remedies are discussed. Hard-copy reporting is used and improved image presentation is considered. New processing algorithms and the development of soft-copy reporting at dedicated workstations are expected in the near future.

Copyright $\odot 1995$ by W.B. Saunders Company

KEY WORDS: digital imaging, mammography, computed radiography.

$\mathbf{T}$ HE DIGITAL ENVIRONMENT offers many potential advantages to the radiologist involved in mammography. The enhancement of the diagnostic features by appropriate image processing, computer-aided diagnosis, soft-copy reporting, and secure data transmission and archiving are the most obvious.

Since 1987 a group of workers at the General Infirmary at Leeds, UK has been investigating the application of digital imaging technology to mammography. The group consists of imaging physicists and computer scientists from FAXIL (the Facility for the Assessment of X-ray Imaging at Leeds) together with engineers, radiographers, and radiologists from the Department of Clinical Radiology.

Initial work involved the digitization of film mammograms, but this was regarded as unlikely to receive wide clinical application because not only does it introduce a further time-consuming process into the production of the final clinical report, but also the quality of the digital image will always be constrained by the quality of the original radiograph. For this reason, direct digital acquisition was regarded as essential and as such, computed radiography (CR) was the only available system. Early work with $\mathrm{CR}^{1}$ had raised serious doubts as to whether the system's relatively low spatial resolution (5 line pairs/ $\mathrm{mm}$ ) was capable of producing mammographic images of clinically acceptable quality. Nevertheless, our own digitization studies suggested that with appropriate image processing, high-quality images could be produced. Consequently, the Leeds Digital Mammography Project was initiated as a collaborative venture between the Leeds team, the UK Department of Health, and Philips Medical Systems Ltd (London, UK), and in June 1990, a Philips Graphic II Series 7000 Computed Radiography System was installed. Series III Fuji HR image plates and level A005 software were used.

\section{CLINICAL PRACTICE}

Initially, all patients presenting for mammography had the mediolateral oblique view performed on CR plates and the craniocaudal, and any additional views, on a conventional filmscreen combination (Fuji HR Mammofine Screens [Fuji Photo Film Co Ltd, Tokyo, Japan] with either Fuji MI-MA or CEA film, CEA Ltd, Milton Keynes, UK). More recently we have moved to total CR mammography.

The majority of patients are symptomatic, although some are screening examinations in high-risk groups. Approximately 2,000 patients are examined a year.

\section{IMAGE QUALITY}

\section{Resolution}

The relatively low resolution of CR systems is a result of a combination of low spatial resolution (5 line pair $/ \mathrm{mm}$ for the high-resolution plates) and a relatively high noise system. Kimme-Smith ${ }^{2}$ in 1992 claimed that CR was unable to detect simulated microcalcifications as large as 280 to $370 \mu \mathrm{m}$, but our own studies have shown particles of similar chemical constitution as small as 160 to $180 \mu \mathrm{m}$ as clearly as conventional film-screen combinations. That this

From the Department of Clinical Radiology, The General Infirmary at Leeds, Leeds, UK.

Supported by the Medical Devices Directorate of the UK Department of Health and Philips Medical Systems Ltd, London, UK.

Address reprint requests to G.J.S. Parkin, FRCR, Department of Clinical Radiology, The General Infirmany at Leeds, Leeds LSI 3EX, UK.

Copyright $\odot 1995$ by W.B. Saunders Company

0897-1889/95/0801-1014\$3.00/0 
spatial resolution is adequate to produce highquality mammographic images is also confirmed by Karssemeijer et al. ${ }^{3}$ Certainly, the increased potential for contrast enhancement inherent in the CR system appears to fully compensate for any reduction in spatial resolution.

\section{Dose Reduction}

Because the dose response of the photostimulable phosphor imaging plate is linear over a very wide range of $x$-ray intensities, the system has tremendous latitude and, hence, the potential for dose reduction. However, as the dose is reduced, the noise in the system increases and consequently we did not reduce the dose rate from that used in conventional mammography.

\section{Image Processing Parameters}

Within the CR system, both contrast processing and spatial frequency processing are used with a total of seven independently variable parameters. Although the equipment is delivered with default settings for each clinical application, it is imperative that in each application, several different combinations of processing parameters are assessed to produce an image in which the reporting radiologist has confidence. In mammography, as well as in other applications of CR, we spent a great deal of time optimizing the processing. When one considers the main diagnostic features of a pathologic mammogram, ie, masses, stromal deformity, and microcalcifications, as well as the demonstration of the normal parenchyma, it is obvious that the optimal demonstration of one feature may well result in the degradation of the image with respect to another. Consequently, the chosen processing parameters are a compromise which must be arrived at with great care. Initially, 15 different sets of processing parameters were examined. By progressively rejecting combinations, we were left with two sets of parameters that were then compared both on clinical images and a specifically designed test object; test object, routine mammography [TOR (MAM)]. ${ }^{4}$ The resulting choice of parameters was then installed as the default setting (GA, 1.2; GT, G; GS, 0.6; RN, 0; RT, P; RE, 1.0). This process of optimization is time consuming, but essential if the full potential of the system is to be achieved.
With these default settings, masses and stromal deformities are well demonstrated. All but the tiniest of microcalcifications are demonstrated and in no case has the inability to show all the microcalcifications evident on the conventional mammogram altered the clinical diagnosis. This has been confirmed both in the routine clinical situation and under test conditions. ${ }^{5}$ The demonstration of the shapes of individual microcalcifications can be significantly improved by reprocessing the image using higher frequency filtration (Fig 1).

\section{RADIOGRAPHIC ASPECTS}

\section{Examination Time}

The total examination time is not increased by using CR because the longer time taken by the $\mathrm{CR}$ unit to produce the finished hard copy ( $3.5 \mathrm{~min}$ ) is balanced by the time it would take to reach and return from the dark room with conventional film-screen cassettes.

\section{Patient Throughput}

The plate-handling capacity of the system ( $\approx 70$ mixed plates $/ \mathrm{hr}$, but $\sim 60$ high-resolution plates/hr) is well within normal symptomatic mammographic practice. Even the busiest symptomatic service rarely exceeds six patients/hour and often has only two to three/hour, so a maximum of 24 to 30 plates/hr is generated. This, incidentally, could be handled by the newer and much cheaper small CR systems. However, our local center of the UK National Breast Cancer Screening Service (Leeds, UK) books women at 4-minute intervals, so the smaller systems would not be able to cope with this throughput.

\section{Staff Training}

Despite the great difference in work practice using the $\mathrm{CR}$ system compared with conventional mammography, all grades of radiographers and radiographic aides rapidly became proficient in its use provided that training was entrusted to a senior designated radiographer with an enhanced level of understanding of the system. 

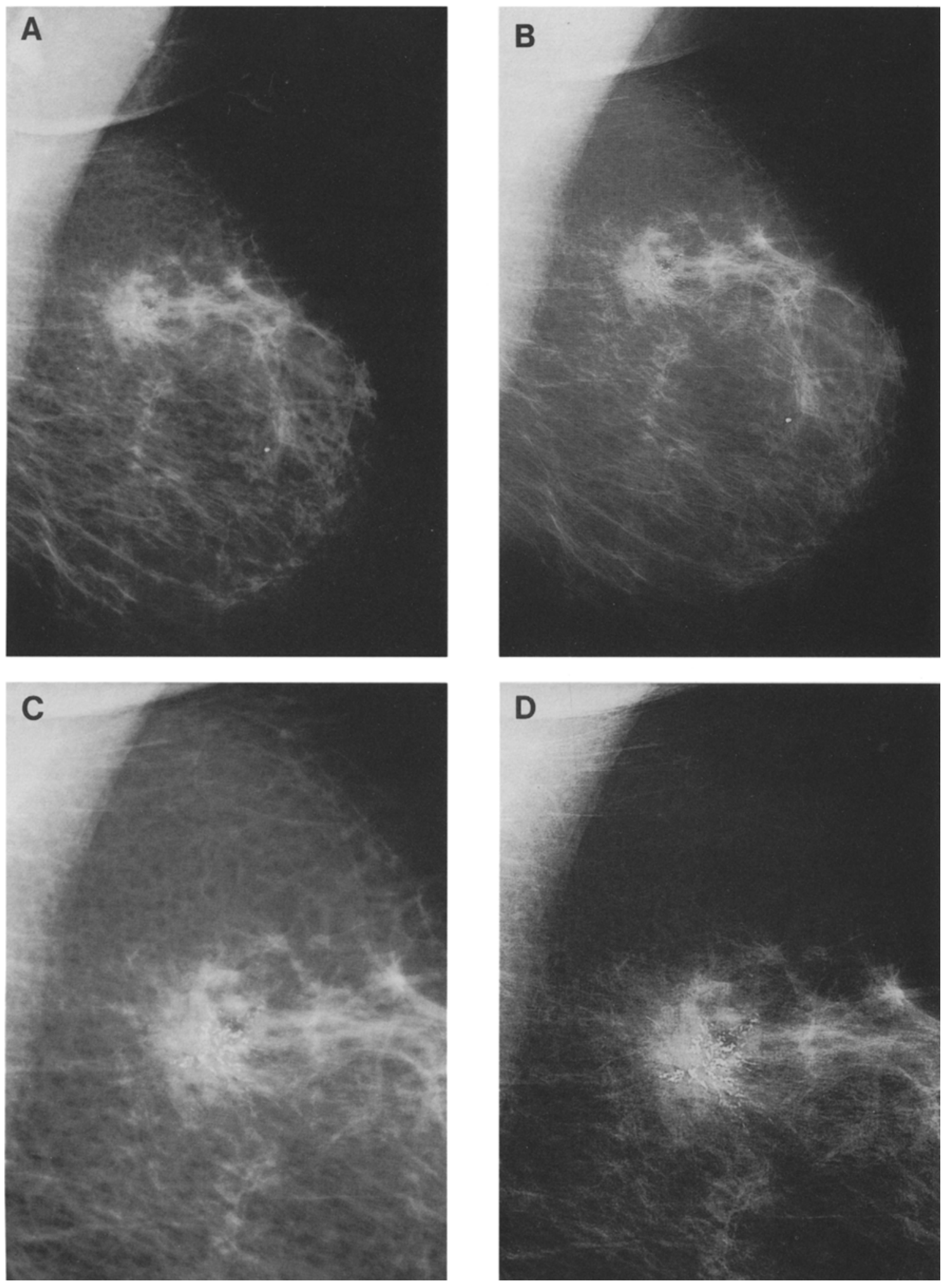

Fig 1. Left mediolateral oblique mammogram with malignant microcalcifications: (A) standard default-processing parameters; (B) with high-frequency filtration; (C) close up, settings as in (A); (D) close up, settings as in (B). 


\section{Problems}

Surprisingly, few problems were encountered in introducing CR mammography to our clinical practice, but three are worthy of being reported.

Dust artifacts. It was noted on some images that clusters of microcalcifications were identified in some projections, but could not be found on any other projection. Eventually we were able to determine that the microcalcifications were, in fact, dust particles adhering to the image plates. In most clinical applications, this would not cause any difficulty, but in mammography, the dust particles are indistinguishable from microcalcifications. The source of the problem was identified as the chimney of the hospital power generating station. Hence, it is necessary to be careful that the $\mathrm{CR}$ reader is installed in a clean environment and also to ensure that the image plates are cleaned, although not as often as conventional screens (twice a day), but much more frequently than CR image plates used for other applications. These latter are usually kept acceptable by the electrostatic cleaner in the image plate reader.

Black films. Initially up to $5 \%$ of images were virtually "black" with no clinically useful data in them. Eventually, this fault was traced to the PRIEF (Pattern Recognizers for Iris of Exposure Field), and because of modification by the manufacturers of the equipment (Fuji) in 1991, this fault has not recurred.

White films. Occasionally, the Exposure Data Recognizer failed to recognize the whole dynamic range of the image or the range was too great for the latitude set and consequently a white film resulted. This could be remedied by extending the latitude of the view menu. When programmed into the system, this worked well, but on upgrading to Series $V$ plates and the corresponding software, it has recurred. Further work is obviously needed on this infrequent, but irritating, problem.

\section{REPORTING}

To date, the clinical service has been conducted using hard-copy reporting. This is currently less than ideal for two particular reasons: hard-copy image presentation and cost.

\section{Hard-Copy Image Presentation}

With the system provided, each hard-copy image is produced on a single sheet of film $\sim 10 \times 14$ in, but the image occupies only $7 \times$ 9.31 in. Because of this, it is impossible to view the image back to back as in conventional mammography (Fig 2), and the unusual film size results in extraneous light around the images on any conventional viewing box.
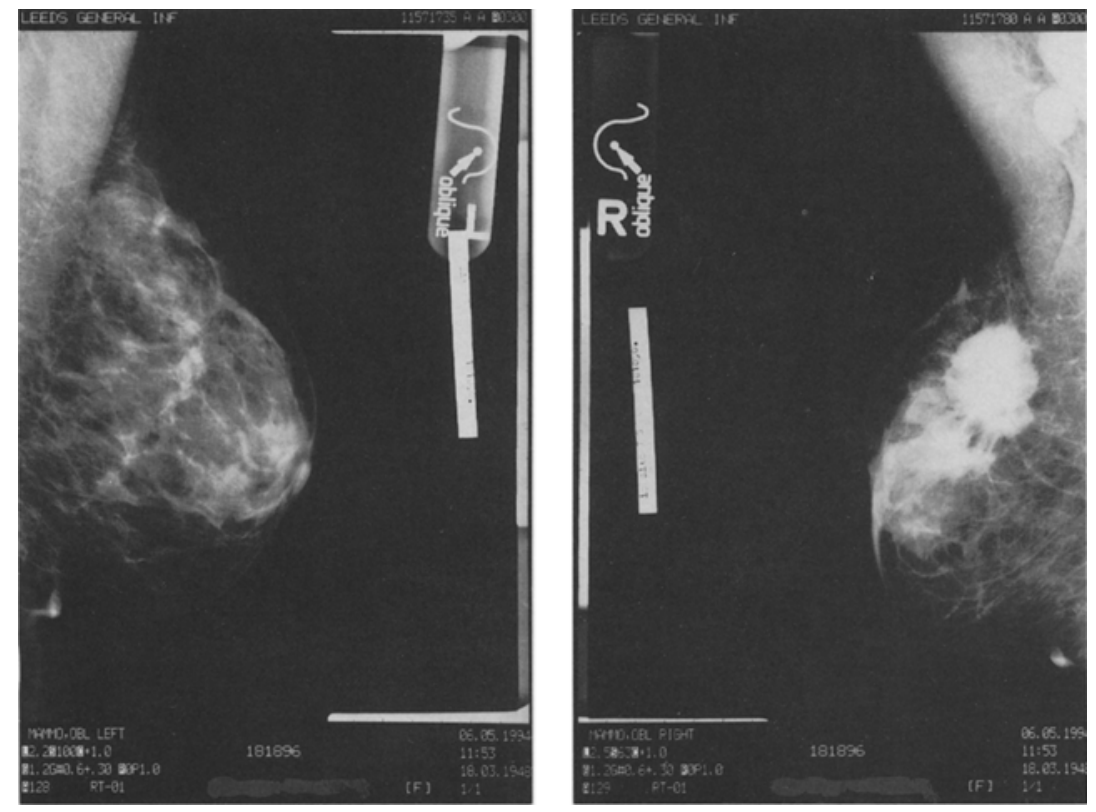

Fig 2. Presentation of hard copy on two $10-\times 14$-in films (large carcinoma of right breast). 


\section{Cost}

In the United Kingdom, conventional mammographic film costs approximately $68 \not \varnothing$ per sheet, but CR film of the above size costs $\$ 1.82$ per sheet. Hence, transfer to total CR more than doubles the film cost. As such, it is difficult to see how direct digital mammography could replace conventional mammography without significant improvements, which fortunately are already becoming available.

\section{IMPROVEMENTS}

\section{Technology}

Already, the Series III image plates have been superceded by Series V with corresponding upgrades in the software. Although we have had them available for an insufficient time to fully evaluate them, subjectively the images are improved.

\section{Hard-Copy Presentation}

The hard-copy format described above is inconvenient and expensive. The upgrade of our system to a Graphic IIe has allowed us to interface the CR system to an Easyvision RAD (Philips Medical Systems Ltd) workstation. This has allowed us to present either two images back to back (Fig 3) or even four images (two obliques and two craniocaudal images) on a single $35-\times 43-\mathrm{cm}$ sheet of laser image film via an existing laser image unit. Such a presentation

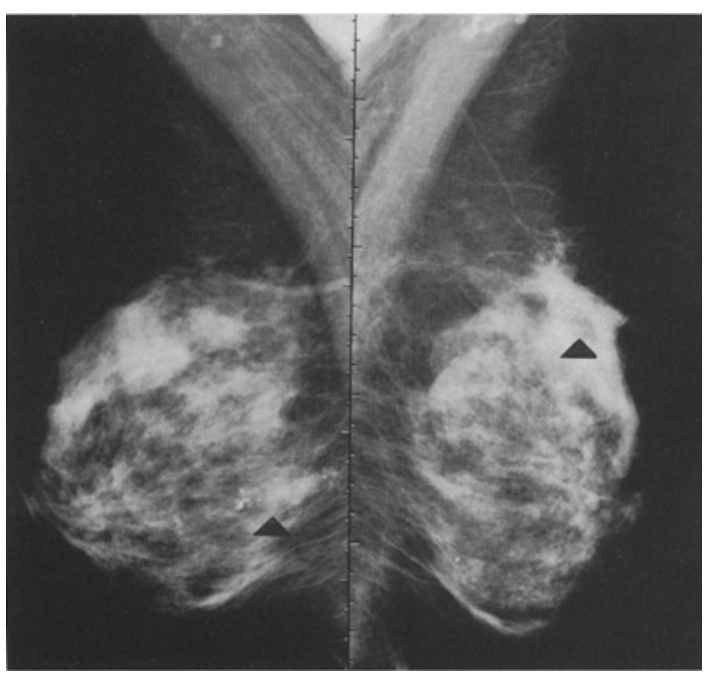

Fig 3. Back-to-back mediolateral oblique images on one film. (Bilateral malignant microcalcification, [arrow].) is not only more convenient, but it is also much less expensive.

\section{New Processing Algorithms}

The ability to deliver the raw digital data to an image processing workstation was initially, in our system, a research tool. This allowed us to develop processing algorithms different from those resident in the CR system. However, the principle is now appearing on the commercial market and potential for alternative processing algorithms is an exciting development in the CR field. Indeed, algorithms developed by our group will be available in commercial products in the near future.

\section{Soft-Copy Reporting}

To date, all reporting has been from hard copy. However, part of our initial research project was the investigation of the feasibility of soft-copy reporting at a custom-built workstation. Such a workstation, based on a PIXAR II imaging computer (ViCom, Fremont, CA) was designed in FAXIL, ${ }^{6}$ and initial trials by the author and colleagues indicated that with a 2,000-line monitor and appropriate image processing, the accuracy of diagnosis was equivalent to hard-copy reporting, but that the time for reporting was increased by approximately $50 \%$. It is expected that the time difference will be reduced with experience. A commercially available version of the reporting workstation is currently being evaluated. Obviously, if strictly controlled trials validate the accuracy of softcopy reporting, the saving in film costs as well as the security and cost effectiveness of the digital archival will be of great significance.

\section{THE FUTURE}

Experience with currently available technology has shown that direct digital mammography can compare favorably with conventional filmscreen mammography. However, even the most ardent enthusiast would admit that current technology does not fulfill all requirements. The ideal digital mammographic unit would have an integrated detector, obviating removal to a separate image reader. The detector would be sealed, excluding dust and other extraneous artifacts. It 
would have an adequate but not over-specified spatial resolution. It would be a low noise system, and, consequently, have a high detective quantum efficiency. The absence of overspecified spatial resolution is, I believe, most important. A current CR mammogram with a $100-\mu \mathrm{m}$ pixel contains $\sim 8$ megabytes of data. Reducing the pixel size to $50 \mu \mathrm{m}$ quadruples the data content. This has implications for data transfer times, storage, and cost. As such, any specification must be adequately justified.

Computer-aided diagnosis, eg, of microcalci- fications and even low-contrast masses, currently engages workers in many centers. The incorporation of such techniques, and even computer diagnosis using neural networks, eg, may eventually be incorporated into the ideal digital mammographic unit.

\section{ACKNOWLEDGMENT}

The author is pleased to acknowledge the support of the Medical Devices Directorate of the United Kingdom Department of Health and Philips Medical Systems (UK) Limited for this work.

\section{REFERENCES}

1. Oestmann JW, Kopans D, Hall DA, et al: A comparison of digital storage phosphors and conventional mammography in the detection of malignant microcalcifications. Invest Radiol 23:725-728, 1988

2. Kimme-Smith $C$ : New and future developments in screen-film mammography equipment and techniques. Radiol Clin North Am 30:55-66, 1992

3. Karssemeijer N, Frieling JT, Hendriks JH: Spatial resolution in digital mammography. Invest Radiol 28:413 419,1993

4. Cowen AR, Brettle DS, Coleman NJ, et al: A prelimi- nary investigation of the imaging performance of photostimulable phosphor computed radiography using a new design of mammographic quality control test object. $\mathrm{Br} \mathrm{J}$ Radiol 65:528-535, 1992

5. Brettle DS, Ward SC, Parkin GJS, et al: A clinical comparison between conventional and digital mammography utilising computer radiography. Br J Radiol 67:464-468, 1994

6. Giles A, Cowen AR, Parkin GJS: A clinical workstation for digital mammography. SPIE Proc 1905:806-817, 1993 